\title{
USE OF INFORMATION INTELLIGENT COMPONENTS FOR THE ANALYSIS OF COMPLEX PROCESSES OF MARINE ENERGY SYSTEMS
}

\author{
Sergei Chernyi \\ Kerch State Maritime Technological University \\ Kerch, Russia, Ordjonikidze, 82 str., 298309 \\ Ph.: +3805005907708, e-mail address: sergiiblack@gmail.com
}

\begin{abstract}
Synchronous motors and their modifications (ac converter-fed motor, etc.) enable to develop low-noise, reliable and economically efficient electric drive systems. The construction of up-to-date systems based on the synchronous machines is impossible without development of the computing software incorporating mathematical and computing simulation. In its turn, modelling of the synchronous machines as a rule is based on the equations by Park-Gorev, application of which requires adoption of a series of allowances. These allowances in a number of cases do not permit to obtain adequate results of the simulation coinciding with the results of field experiments of the systems under review. Moreover, while applying the system of equations by Park-Gorev to research the systems including interaction of synchronous machines with semiconducting converters of electric energy it is necessary simulate the process of formation their controlling signals in the sphere of frequency. If the states of converter's keys are defined not only by controlling impulses but also by the state of currents in the synchronous machines flowing through them, such approach is not reasonable.
\end{abstract}

Keywords: power systems, intellectual systems, synchronous machines, ship, simulation

\section{Introduction}

Synchronous machines as compared with asynchronous ones with the same capacity have higher efficiency, less dependence of electromagnetic torque on the power supply voltage; the frequency of their revolution does not depend on the load of the shaft. Synchronous machines are applied to compensate reactive capacity of the circuit, etc.

On the other side, due to the short-term reduction in supply voltage as well as in case of loss of excitation there is capability to change the mode of a synchronous machine into a generator or asynchronous mode of operation. As synchronous motors are consumers of the first category, it may be the cause of significant economic losses, risks for human health and life.

At present, tasks of modelling of electric mechanical systems in synchronous machines are solved in specialized software such as Mustang, DAKAR, ANARES-2000, EUROSTAG, etc. These types of software make possible to model synchronous machines based on equations by Park-Gorev taking into account the damper contours. Table 1 provides certain specific features of modelling in the abovementioned systems.

Table 1. Specific features of models of the synchronous machines in the widespread programs

\begin{tabular}{|l|l|}
\hline Program & Specific feature of the model used \\
\hline DAKAR & Dependence of synchronous capacitances of the machine on frequency is not taken into account \\
\hline ANARES-2000 & Changes in frequency of revolutions are registered \\
\hline Mustang & Current displacement in the damper contours is evaluated roughly by an adjusting rate \\
\hline EUROSTAG & $\begin{array}{l}\text { Saturation is evaluated by applying Shakshaft's model. Currents of the machine depend on the } \\
\text { circuit frequency. Mechanical characteristics of the generator are constructed by means of the } \\
\text { equation of the revolving motion dynamics. }\end{array}$ \\
\hline
\end{tabular}

In the models implemented in all the represented programs the following allowances are made (Bolotin and Vainer, 1974):

a) modelling is made in the system of coordinates d-q;

b) saturation and hysteresis of the magnetizing characteristics are not taken into account;

c) an effect of the current displacement is not taken into account or is taken into account approximately via relevant coefficients;

d) a symmetry of the stator windings is not taken into account (Power System Harmonic, 1984); 
e) a field winding is considered uniaxial;

f) a damper winding is reduced to two squirrel cages with perpendicular axes.

The similar specific features have models included into the program Simulink in the medium MATLAB (Chernyi and Zhilenkov, 2015).

While researching systems based on synchronous machines modelling the system of the automatic voltage regulation (AVR) and a number of other systems for the automatic regulation is required. In the programs under review models are designed to control the excitation current of the models of synchronous generators; there is no minor loop of control for excitation current; there is no possibility to realize PID-regulator in the primary voltage contour and excitation contour: there is no possibility to simulate field suppression by means of regeneration of energy into the circuit (Govindarajan, 1991).

These limitations in a number of cases make unpractical to apply the abovementioned programs in order to model the systems of excitation of synchronous machines.

Moreover, existing models as a rule do not allow obtaining adequate results if necessary to take into account limitation of capacity of a synchronous generator, absence of a neutral conductor (according to the safety requirements in most autonomous plants it is necessary to apply systems with isolated neutral) or power supply of synchronous motor, that is a specific feature of any autonomous electric power system (Manchur and Erven, 1992).

\section{Setting of the problem of a synchronous machine simulation}

Figure 1 gives elemental composition of power plant used on vessels, drilling platforms, etc. and Figure 2 demonstrates elements of the power propulsion plant based on the synchronous motor. In autonomous submersible apparatuses, the composition represented on Figure 2 will have a bank of batteries as a power supply and the power supply of the synchronous motor will be formed via the autonomous invertor (frequency invertor). In all other aspects, the schemes will be the same.

All the elements by their performance characteristics will be divided into linear, linearized and nonlinear ones. Linear elements are described by means of pure first-degree equations (both algebraic and differential ones) (Tolshin et al., 1969). We refer to linearized elements purely described by non-linear equations but with slight deviations, they may be converted into linear equations by means of linearization, error obtained in description almost not affecting the performance parameters. Non-linear elements are those elements, linearization of which is practically impossible due to the obtained error in performance parameters after applying the procedure of linearization is unacceptably large (Konstantinov, 1988).

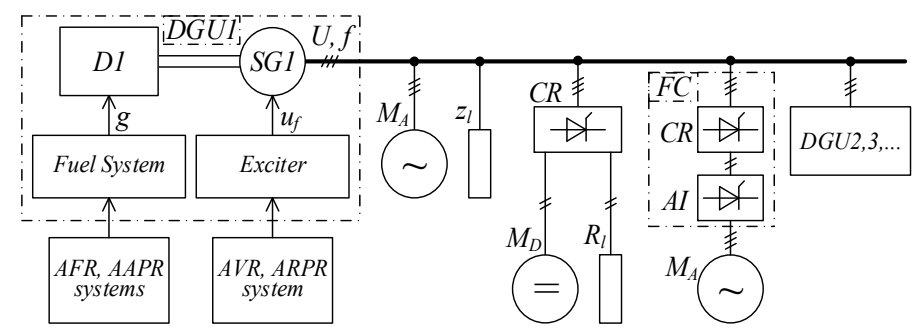

Figure 1. Generalized functional scheme of the ship's electric power plant: DGUn - diesel-generator unit (diesel Dn + generator $\mathrm{SGn}$, where $\mathrm{n}$ - is a number of DGU); AFR, AVR, AAPR and ARPR - systems of automated regulation of frequency, voltage, active and reactive capacity of a synchronous generator; MA and MD - ac and dc motors; $\mathrm{zl}$ and Rl - resistance of static load on ac and dc; CR - controlled rectifier; AI - autonomous inverter (of voltage or current); FC - frequency converter

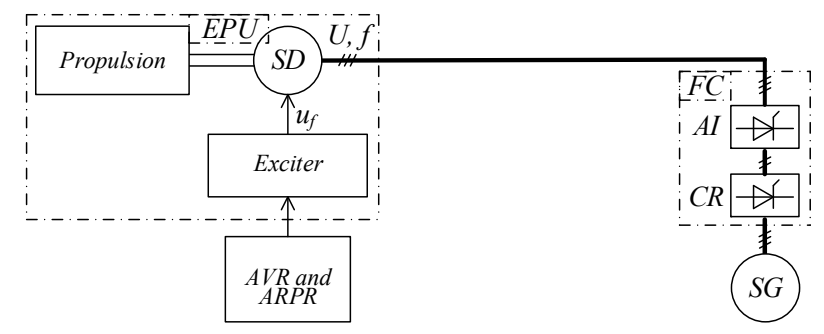

Figure 2. Generalized functional scheme of a ship's propulsion plant with the element of the electric power plant 
Of described in Figures 1 and 2 elements the controlled rectifier CR and autonomous inverter AI may be referred to non-linear ones. The specific features of non-linear description of the mentioned elements are as follows (Zhilenkov and Chernyi, 2015):

1) non-linearity of a relay type;

2) non-linearity parameters depend on time that allows referring them to non-autonomous type;

3) there are not less than three non-linearities in each controlled rectifier CR and autonomous inverter AI (for three-phase electric mains).

Thus, by the type of transitional and settled processes the systems under review are referred to nonlinear ones. Methods of analysis and synthesis of such systems are most comprehensively developed in the section of non-linear systems for the theory of automated control (TAC). The significant specific features of non-linear systems are as follows:

- non-implementation of the superposition principle and the fact that research of the non-linear system with several impacts should not be reduced to research with one impact;

- stability and the character of the transitional process depends on the value of the initial deviation from the equilibrium position;

- with fixed external impacts several equilibrium positions (and sometimes even infinite aggregate) are possible;

- there emerge free settled processes which are impossible in non-linear systems (for example, autofluctuations).

Considering these specific features, resulting in complex calculations, one is limited by one or two non-linearities in one estimated system, and not more. In spite of great efforts to increase the efficiency of the calculations of non-linear systems, it can be stated that at present there are no universal analytical (mathematical) methods of research on non-linear systems.

All the known methods for calculations of non-linear systems, which are significant in selecting one's own trends in research, have the following specific features (Singh et al., 1999):

1) a set of signals, referring to a non-linear link, is not undergone by any changes that in particular allows to use initial simplest forms of non-linear description of these links;

2) folding of structural schemes made of non-linear links as it is almost impossible for schemes divided by linear links and for neighbouring links it results in unreasonably complicated mathematical description;

3) a linear portion of the system transforms optionally by the criterion of productivity of calculations on the condition that all the signals by which linear part is connected with non-linear links remain unchanged (Power System Harmonics, 1983).

In models of the systems based on synchronous machines (SM) the model like the system is applied. If operating SM as a generator for a linear load such a model has a lot of advantages: the number of differential equations are minimum: 5 - if to take into account only electrical processes and 7 - if to take into account electromechanical; models of voltage regulation through the excitation winding circuit are the simplest, as PGE are connected to the excitation winding; with several simple allowances derivation of analytical expressions for the mode of short circuit SM and voltage SM, etc. is possible.

Modelling of SM together with semiconductor converters operation by means of equations by ParkGorev is not assumed productive for the following reasons (Chernyi and Zhilenkov, 2015; Konoplev, 2008):

1. Each phase winding of the SM stator is switched in its non-linearity formed by the scheme of the phase of controlled converter (CR, $\mathrm{AI})$. Reduction of equations of three windings of a stator to a rotor undertaken by two steps firstly applying liner conversion of phases (signals of three-phase winding of a stator in axes A-B-C to signals of two-phase winding of a stator in fixed axes $\alpha-\beta$ ) and then non-linear conversion of coordinates (signals of two-phase winding of a stator in fixed axes $\alpha-\beta$ into signals of twophase stator winding in axes d-q, associated with excitation winding of the SM) converses non-linearly three-six (3 - for zero scheme of a rectifier and 6 - for a bridge circuit) non-linearities, for example, CR into two giant non-linearities in circuits of primary windings by axes d-q.

2 . In the model SM there will be three-six initially non-linear links caused by CR and non-linearities of coordinate conversions from $\alpha-\beta$ into $d-q$ are added to them.

3. Non-linear links ( 3 or 6 ) pass through conversions, which are analytical forms of the structural changes of the connection of non-linear links in the system.

4. Input and output signals of conversed non-linear links do not coincide with signals, by which the state of a thyristor $V S$ (diode) $C R$, - voltage $u_{V S}$, current $i_{V S}$ is defined.

From the abovementioned reasons it is seen that modelling of the synchronous machine operating with the semiconducting converter of the electric power, in sphere of leads to the problems which are not only solved in the theory of automated control but it is considered reasonable not to be created. Thus, in the 
model of the system operating together with a semiconducting converter of the electric power, we have to reject the model of a synchronous machine by equations by Park-Gorev and apply the model with the natural phase signals of the primary windings of the synchronous machine - voltages $u_{A}, u_{B}, u_{C}$ and currents $i_{A}, i_{B}, i_{C}$. This paper is devoted to the development of such a model.

\section{SM model in the axes of stator windings}

The scheme of the loaded SG without a zero terminal and natural windings on the stator and rotor is given in Fig. 3.

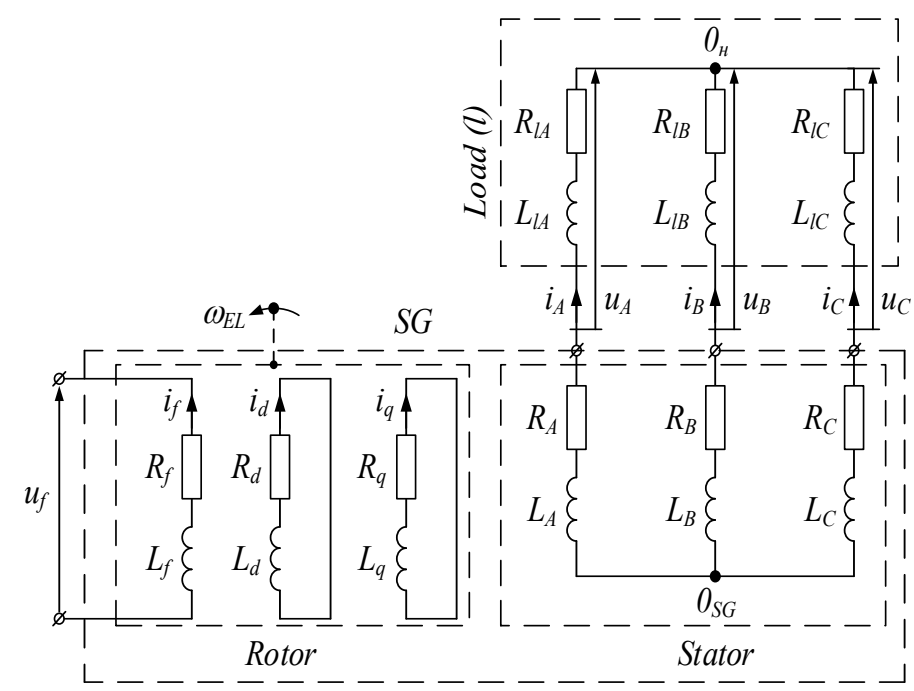

Figure 3. SM scheme with physical primary and secondary windings

In this scheme in all the windings of the synchronous machine physical currents $i_{\mathrm{A}}, i_{B}, i_{C}, i_{f}, i_{d}$ and $i_{q}$ flow, and the rotor revolves as for the stator with the frequency $\omega_{e l}$. SM is described by the system of equations (1) (Chernyi and Zhilenkov, 2015; Lokhanin et al., 2008; Markovich, 1969):

$$
\left\{\begin{array}{l}
i_{A}+i_{B}+i_{C}=0, \\
u_{A}=\left(R_{A}+R_{i \dot{A}}\right) i_{A}+p \Psi_{A}, \\
u_{B}=\left(R_{B}+R_{i B}\right) i_{B}+p \Psi_{B}, \\
u_{C}=\left(R_{C}+R_{i C}\right) i_{C}+p \Psi_{C}, \\
u_{f}=R_{f} \cdot i_{f}+p \Psi_{f}, \\
0=R_{d} \cdot i_{d}+p \Psi_{d}, \\
0=R_{q} \cdot i_{q}+p \Psi_{q}, \\
\Psi_{A}=\left(L_{A}+L_{i A}\right) i_{A}+L_{A B} i_{B}+L_{A C} i_{C}+L_{A f} i_{f}+L_{A d} i_{d}+L_{A q} i_{q}, \\
\Psi_{B}=L_{A B} i_{A}+\left(L_{\hat{A}}+L_{i \hat{A}}\right) i_{B}+M_{B C} i_{C}+L_{B f} i_{f}+L_{B d} i_{d}+L_{B q} i_{q}, \\
\Psi_{C}=L_{A C} i_{A}+L_{B C} i_{B}+\left(L_{\tilde{N}}+L_{i \tilde{N}}\right) i_{C}+L_{C f} i_{f}+L_{C d} i_{d}+L_{C q} i_{q}, \\
\Psi_{f}=L_{A f} i_{A}+L_{B f} i_{B}+L_{C f} i_{C}+L_{f} i_{f}+L_{f d} i_{d}+L_{f q} i_{q}, \\
\Psi_{d}=L_{A d} i_{A}+L_{B d} i_{B}+L_{C d} i_{C}+L_{f d} i_{f}+L_{d} i_{d}+L_{d q} i_{q}, \\
\Psi_{q}=L_{A q} i_{A}+L_{B q} i_{B}+L_{C q} i_{C}+L_{f q} i_{f}+L_{d q} i_{d}+L_{q} i_{q} .
\end{array}\right.
$$


The system of 13 equations includes one independent variable - excitation voltage $u f$ - and 12 variables of the state - currents $i_{A}, i_{B}, i_{C}, i_{f}, i_{d}, i_{q}$ and current linkage $\Psi_{A}, \Psi_{B}, \Psi_{C}, \Psi_{f}, \Psi_{d}, \Psi_{q}$. The required condition of solvability of the system of equations is realized.

After calculation of phase currents $i_{A}, i_{B}, i_{C}$ the phase voltages may be estimated by expressions (2).

$$
\left\{\begin{array}{l}
u_{A}=\left(p L_{i \grave{A}}+R_{i \grave{A}}\right) i_{A}, \\
u_{B}=\left(p L_{i B}+R_{i B}\right) i_{B}, \\
u_{C}=\left(p L_{i C}+R_{i C}\right) i_{A} .
\end{array}\right.
$$

However, self-inductances (with single-letter index) and mutual inductances (with double letter index) $L$ in equations (1) are periodical coefficients from the angle of turn $\varphi_{e l}$ of the rotor respectively the stator. There is no information in catalogue data SM for transient values $L$. It is used to include in the catalogue data SM data by inductances $L$, which are suitable for model SM by Park-Gorev's equations. For this model, inductances are the constant values. Such feature of the constancy L in the model by ParkGorev's equation is reached due to that all the windings of the SM being connected to the field winding are mutually non-rotatable in respect of each other (Mikhalev, 2013).

If to set an objective to produce SM model, in which physical signals of the stator should be obviously present, i.e. phase voltages $u_{A}, u_{B}, u_{C}$ and currents $i_{A}, i_{B}, i_{C}$, and parameters of the SM should be constant and allow to make calculations via catalogue data of SG, it is necessary:

1) to set rotor windings to stator windings that makes them mutually non-rotatable and allows apply constant values $L$;

2) to produce formulas for re-calculation of catalogue values $L$ into values $L$ in the model based on stator windings.

Let us make the system of equations describing synchronous generator as two-phase machine in the optional coordinate axes $u-v$, being general for all the SM windings (both stator and rotor) and rotating in the space with a random velocity $\omega_{K}$.

In such system, the stator will be described by means of the following equations:

$u_{1 u}=R_{1} \cdot i_{1 u}+p \Psi_{1 u}-\omega_{K} \cdot \Psi_{1 v}$,

$u_{1 v}=R_{1} \cdot i_{1 v}+p \Psi_{1 v}+\omega_{K} \cdot \Psi_{1 u}$,

where $R_{I}$ - armature resistance, which with the symmetrical stator is equal to resistance $R_{A}, R_{B}$ and $R_{C}$ of any stator winding.

For field winding:

$u_{f u}=R_{f} \cdot i_{f u}+p \Psi_{f u}-\left(\omega_{K}-\omega_{e l}\right) \cdot \Psi_{f v}$,

$u_{f v}=R_{f} \cdot i_{f v}+p \Psi_{f v}+\left(\omega_{K}-\omega_{e l}\right) \cdot \Psi_{f u}$

where $\omega_{e l}$ - the velocity of rotation of rotor windings respectively stator SM.

For short-circuited damper winding:

$0=R_{2} \cdot i_{2 u}+p \Psi_{2 u}-\left(\omega_{K}-\omega_{e l}\right) \cdot \Psi_{2 v}$,

$0=R_{2} \cdot i_{2 v}+p \Psi_{2 v}+\left(\omega_{K}-\omega_{e l}\right) \cdot \Psi_{2 u}$

where $R_{2}$ - true resistance of a damper winding which with the symmetrical construction is equal to any resistance $R_{d}$ and $R_{q}$ (Fig.3).

As a result, we obtain a system of equations (3), which describes SM as a two-phase machine in optional axes $u-v$, rotating with the velocity $\omega_{K}$.

In such mathematical description SM with each velocity $\omega_{K}$ of the inductance $L$ and mutual inductance M, by means of which flux linkages $\Psi_{l u}, \Psi_{l v}, \Psi_{f u}, \Psi_{f v}, \Psi_{2 u}, \Psi_{2 v}$ are calculated, will be constant values as all the windings in the SM model are mutually non-rotatable. Moreover, it is very important, in every choice of coordinate axes $u-v$ and frequency of their rotation $\omega_{K}$ values $L$ remain unchangeable and only frequency of voltages $u_{l u}, u_{l v}, u_{f u}, u_{f v}$ change.

Let us assume coordinate axes $u-v$ superimposed with axes $d-q$ of the field winding. Thus, frequency of their rotation $\omega_{K}$ will be equal to frequency of rotation $\omega_{e l}$ of the rotor (Fig.3). After plugging $\omega_{K}=\omega_{e l}$ in the system (3) changes of indices $u \rightarrow d$ and $v \rightarrow q$ we obtain the system (4): 


$$
\begin{aligned}
& \left\{\begin{array}{l}
u_{1 u}=R_{1} \cdot i_{1 u}+p \Psi_{1 u}-\omega_{K} \cdot \Psi_{1 v}, \\
u_{1 v}=R_{1} \cdot i_{1 v}+p \Psi_{1 v}+\omega_{K} \cdot \Psi_{1 u}, \\
u_{f u}=R_{f} \cdot i_{f u}+p \Psi_{f u}-\left(\omega_{K}-\omega_{e l}\right) \cdot \Psi_{f v}, \\
u_{f v}=R_{f} \cdot i_{f v}+p \Psi_{f v}+\left(\omega_{K}-\omega_{e l}\right) \cdot \Psi_{f u}, \\
0=R_{2} \cdot i_{2 u}+p \Psi_{2 u}-\left(\omega_{K}-\omega_{e l}\right) \cdot \Psi_{2 v}, \\
0=R_{2} \cdot i_{2 v}+p \Psi_{2 v}+\left(\omega_{K}-\omega_{e l}\right) \cdot \Psi_{2 u} .
\end{array}\right. \\
& \left\{\begin{array}{l}
u_{1 d}=R_{1} \cdot i_{1 d}+p \Psi_{1 d}-\omega_{e l} \cdot \Psi_{1 q}, \\
u_{1 q}=R_{1} \cdot i_{1 q}+p \Psi_{1 q}+\omega_{e l} \cdot \Psi_{1 d}, \\
u_{f}=R_{f} \cdot i_{f}+p \Psi_{f}, \\
0=R_{2} \cdot i_{2 d}+p \Psi_{2 d}, \\
0=R_{2} \cdot i_{2 q}+p \Psi_{2 q},
\end{array}\right.
\end{aligned}
$$

In the system (4) the equation for field winding by the quadrature axis $q$ is omitted as such winding is almost absent and in the equation of the field winding by the direct axis d index $\mathrm{d}$ is also omitted.

System (4) is a system of the Park-Gorev's equation. In order to calculate its flux linkages SM catalogue data may be used.

Let us take coordinate axes $u-v$ superimposed with axes $\alpha-\beta$, moreover axis $\alpha$ coincides with axis $A$ of the non-rotating in the space winding of stator phase A. It is natural that the frequency of rotation $\omega_{K}$ of the coordinate axes $\alpha-\beta$ will be equal to zero. After plugging $\omega_{K}=0$ in the system (3) and changes of indices $\mathrm{u} \rightarrow \alpha$ and $\nu \rightarrow \beta$ we obtain:

$$
\left\{\begin{array}{l}
u_{1 \alpha}=R_{1} \cdot i_{1 \alpha}+p \Psi_{1 \alpha}-\omega_{e l} \cdot \Psi_{1 q}, \\
u_{1 \beta}=R_{1} \cdot i_{1 \beta}+p \Psi_{1 \beta}+\omega_{e l} \cdot \Psi_{1 d}, \\
u_{f \alpha}=R_{f} \cdot i_{f \alpha}+p \Psi_{f \alpha}+\omega_{e l} \cdot \Psi_{f \beta}, \\
u_{f \beta}=R_{f} \cdot i_{f \beta}+p \Psi_{f \beta}-\omega_{e l} \cdot \Psi_{f \alpha}, \\
0=R_{2} \cdot i_{2 \alpha}+p \Psi_{2 \alpha}+\omega_{e l} \cdot \Psi_{2 \beta}, \\
0=R_{2} \cdot i_{2 \beta}+p \Psi_{2 \beta}-\omega_{e l} \cdot \Psi_{2 \alpha} .
\end{array}\right.
$$

Flux linkages of the SM windings in axes $\alpha-\beta$ will be represented as follows:

$$
\left\{\begin{array}{l}
\Psi_{1 \alpha}=L_{1} \cdot i_{1 \alpha}+L_{1 f} i_{f \alpha}+L_{12} i_{2 \alpha}, \\
\Psi_{1 \beta}=L_{1} \cdot i_{1 \beta}+L_{1 f} i_{f \beta}+L_{12} i_{2 \beta}, \\
\Psi_{f \alpha}=L_{1 f} \cdot i_{1 \alpha}+L_{f} i_{f \alpha}+L_{2 f} \cdot i_{2 \alpha}, \\
\Psi_{f \beta}=L_{1 f} \cdot i_{1 \beta}+L_{f} i_{f \beta}+L_{2 f} \cdot i_{2 \beta}, \\
\Psi_{2 \alpha}=L_{12} \cdot i_{1 \alpha}+L_{2 f} i_{f \alpha}+L_{2} \cdot i_{2 \alpha}, \\
\Psi_{2 \beta}=L_{12} \cdot i_{1 \beta}+L_{2 f} i_{f \beta}+L_{2} \cdot i_{2 \beta} .
\end{array}\right.
$$

Inductances in the system (6) coincide with the catalogue values for SM, if in the equations of the system (5) relative values for voltages and currents, which are already set to the stator windings, are applied.

SM model as a system of equations (5) have fictitious voltages $u_{l \alpha}, u_{1 \beta}$ and currents $i_{l \alpha} i_{l \beta}$ of the double-winding stator in axes $\alpha-\beta$. As to model semiconducting converter supplying from SG it is required to operate by true voltages $u_{A}, u_{B}, u_{C}$ and currents $i_{A}, i_{B}, i_{C}$, in the stator circuit, so from the system (5), describing two-phase machine it is necessary to pass to three-phase machine by stator. While passing from the two-phase stator to three-phase one, we solve the task of conversation of stator variables of the equivalent two-phase machine in the orthogonal coordinates $\alpha-\beta$ into true variables of the stator for the three-phase machine in axes $A-B-C$. Formulas for the direct conversion of currents $i_{1 \alpha}, i_{1 \beta}$ into currents $i_{A}, i_{B}, i_{C}$, obtained from the condition of invariance of SG capacity, estimated in axes $\alpha$ - $\beta$ and $A-B-C$, are as follows (7): 
$\left\{\begin{array}{c}i_{A}=\sqrt{\frac{2}{3}} i_{1 \alpha} \\ i_{B}=\sqrt{\frac{1}{6} i_{1 \alpha}+\sqrt{\frac{1}{2}} i_{1 \beta}} \\ i_{C}=-\sqrt{\frac{1}{6} i_{1 \alpha}}-\sqrt{\frac{1}{2} i_{1 \beta}}\end{array} \quad\right.$ or $\quad\left|\begin{array}{ll}i_{A} \\ i_{B} \\ i_{C}\end{array}\right|=\left|\begin{array}{rr}\sqrt{\frac{1}{2}} & 0 \\ \sqrt{\frac{1}{6}} & \sqrt{\frac{1}{2}} \\ -\sqrt{\frac{1}{6}} & -\sqrt{\frac{1}{2}}\end{array}\right| \cdot \mid \begin{array}{l}i_{1 \alpha} \mid \\ i_{1 \beta} \mid\end{array}$

Formulas for the inverse conversion of currents (8) $i_{A}, i_{B}, i_{C}$ into currents $i_{1 \alpha}, i_{1 \beta}$, obtained from the condition of invariance of SG capacity, are inverse to formulas (7):

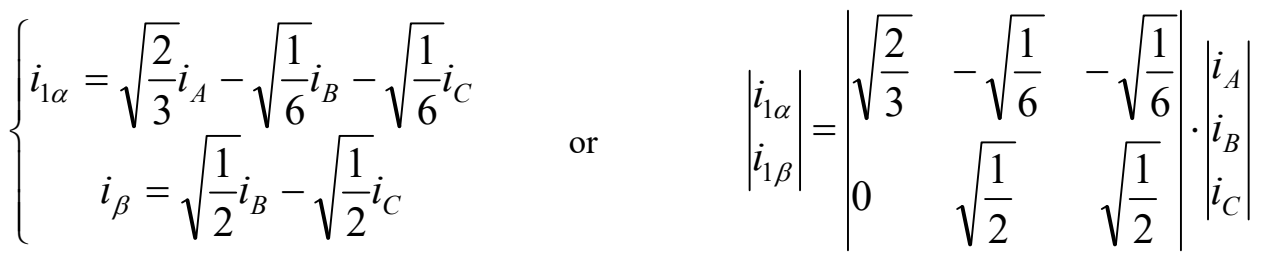

And taking into account congruence $i_{A}+i_{B}+i_{C}=0$ they are as follows (9):

$\left\{\begin{array}{c}i_{1 \alpha}=\sqrt{\frac{3}{2} i_{A}} \\ i_{\beta}=\sqrt{\frac{1}{2} i_{B}-\sqrt{\frac{1}{2} i_{C}}}\end{array} \quad\right.$ or $\quad\left|\begin{array}{l}i_{1 \alpha} \\ i_{1 \beta}\end{array}\right|=\left|\begin{array}{ccc}\sqrt{\frac{2}{3}} & 0 & 0 \\ 0 & \sqrt{\frac{1}{2}} & -\sqrt{\frac{1}{2}}\end{array}\right| \cdot \mid \begin{array}{c}\left|\begin{array}{c}i_{A} \\ i_{B} \\ i_{C}\end{array}\right|\end{array}$

Formulas for conversion of voltages $u_{A}, u_{B}, u_{C}$ and $u_{1 \alpha}, u_{1 \beta}$ are the same as formulas (7)-(9).

Let us substitute into the first two equations of the system (5) expressions of flux linkages $i_{1 \alpha}, i_{1 \beta}$ , taken from (9). Let us make in equations of the system (5) and expressions of flux linkages (6) substitutions of currents $i_{1 \alpha}, i_{1 \beta}$ and voltages $u_{1 \alpha}, u_{1 \beta}$, represented in axes $\alpha-\beta$, for currents $i_{A}, i_{B}, i_{C}$ and voltages $u_{A}, u_{B}, u_{C}$, represented in axes $A-B-C$, applying formulas (9). We obtain system of SM equations (10) and expressions for flux linkages (11).

$$
\left\{\begin{array}{c}
u_{A}=\left(R_{1}+p L_{1}\right) i_{A}+\sqrt{\frac{2}{3}} p L_{12}\left(i_{2 \alpha}+i_{f \alpha}\right) \\
u_{B}=\left(R_{1}+p L_{1}\right) i_{B}+\frac{1}{\sqrt{2}} p L_{12}\left(i_{2 \beta}+i_{f \beta}\right)-\frac{1}{\sqrt{6}} p L_{12}\left(i_{2 \alpha}+i_{f \alpha}\right) \\
u_{C}=\left(R_{1}+p L_{1}\right) i_{C}-\frac{1}{\sqrt{2}} p L_{12}\left(i_{2 \beta}+i_{f \beta}\right)-\frac{1}{\sqrt{6}} p L_{12}\left(i_{2 \alpha}+i_{f \alpha}\right) \\
U_{2 \alpha}=R_{2} i_{2 \alpha}+p L_{2} i_{2 \alpha}+p L_{12}\left(\sqrt{\frac{3}{2}} \cdot i_{A}+i_{f \alpha}\right)+\omega_{\dot{Y} \tilde{E}}\left(L_{2} i_{2 \beta}+L_{12} \frac{1}{\sqrt{2}} \cdot\left(i_{B}-i_{C}\right)+L_{12} i_{f \beta}\right) \\
U_{2 \beta}=R_{2} i_{2 \beta}+p L_{2} i_{2 \beta}+p L_{12}\left(\frac{1}{\sqrt{2}} \cdot\left(i_{1 B}-i_{1 C}\right)+i_{f \beta}\right)-\omega_{\dot{Y} \tilde{E}}\left(L_{2} i_{2 \alpha}+L_{12} \sqrt{\frac{3}{2}} \cdot i_{A}+L_{12} i_{f \alpha}\right) \\
U_{f \alpha}=R_{f} i_{f \alpha}+p L_{f} i_{f \alpha}+p L_{12}\left(i_{2 \alpha}+\sqrt{\frac{3}{2}} \cdot i_{A}\right)+\omega_{\dot{Y} \ddot{E}}\left(L_{f} i_{f \beta}+L_{12} i_{2 \beta}+L_{12} \frac{1}{\sqrt{2}} \cdot\left(i_{B}-i_{C}\right)\right) \\
U_{f \beta}=R_{f} i_{f \beta}+p L_{f} i_{f \beta}+p L_{12}\left(i_{2 \beta}+\frac{1}{\sqrt{2}} \cdot\left(i_{B}-i_{C}\right)\right)-\omega_{\dot{Y} \ddot{E}}\left(L_{f} i_{f \alpha}+L_{12} i_{2 \alpha}+L_{12} \sqrt{\frac{3}{2}} \cdot i_{A}\right)
\end{array}\right.
$$


$\left|\begin{array}{l}\Psi_{A} \\ \Psi_{B} \\ \Psi_{C} \\ \Psi_{2 \alpha} \\ \Psi_{2 \beta} \\ \Psi_{f \alpha} \\ \Psi_{f \beta}\end{array}\right|=\left|\begin{array}{ccccccc}L_{1} & 0 & 0 & \sqrt{\frac{2}{3}} L_{12} & 0 & \sqrt{\frac{2}{3}} L_{12} & 0 \\ 0 & L_{1} & 0 & -\frac{1}{\sqrt{6}} L_{12} & \frac{1}{\sqrt{2}} L_{12} & -\frac{1}{\sqrt{6}} L_{12} & \frac{1}{\sqrt{2}} L_{12} \\ 0 & 0 & L_{1} & -\frac{1}{\sqrt{6}} L_{12} & -\frac{1}{\sqrt{2}} L_{12} & -\frac{1}{\sqrt{6}} L_{12} & -\frac{1}{\sqrt{2}} L_{12} \\ 0 & \frac{1}{\sqrt{2}} L_{12} & -\frac{1}{\sqrt{2}} L_{12} & 0 & L_{2} & 0 & L_{12} \\ \frac{\sqrt{\frac{3}{2}} L_{12}}{0} & 0 & 0 & L_{2} & 0 & L_{12} & 0 \\ \sqrt{\frac{3}{2}} L_{12} & 0 & 0 & L_{12} & 0 & L_{f} & 0 \\ 0 & \frac{1}{\sqrt{2}} L_{12} & -\frac{1}{\sqrt{2}} L_{12} & 0 & L_{12} & 0 & L_{f}\end{array}\right| \cdot\left|\begin{array}{c}i_{A} \\ i_{B} \\ i_{C} \\ i_{2 \alpha} \\ i_{2 \beta} \\ i_{f \alpha} \\ i_{f \beta}\end{array}\right|$

We describe the mechanical portion of the SM model by equations (12).

$$
\begin{aligned}
\Delta \omega(t) & =\frac{1}{2 J} \int_{0}^{t}\left(M_{\grave{I} \AA \tilde{O}}-M_{e l}\right) d t \\
\omega_{e l}(t) & =\Delta \omega(t)+\omega_{S}
\end{aligned}
$$

where $\Delta \omega(t)$ - deviation of the angular frequency of rotor revolution from the synchronous one; $\mathrm{J}$ - rotor moment of inertia; $M_{M E X}$ - momentum; $M_{e l}$ - electromagnetic torque; damping ratio; $\omega_{e l}(t)$ - angular frequency of rotor revolution; $\omega_{S}$ - synchronous angular frequency of revolution.

Uniting the systems (10), (11) and (12), we obtain the comprehensive system of equations (13), describing SM in the non-rotating axes, applying catalogue parameters and operating with true currents and voltages of the stator circuit (three-phase machine by stator).

$$
\left\{\begin{array}{c}
u_{A}=R_{1} i_{A}+p \Psi_{A} \\
u_{B}=R_{1} i_{1 B}+p \Psi_{B} \\
u_{C}=R_{1} i_{1 C}+p \Psi_{C} \\
U_{2 \alpha}=R_{2} i_{2 \alpha}+p \Psi_{2 \alpha}+\omega_{e l} \Psi_{2 \beta} \\
U_{2 \beta}=R_{2} i_{2 \beta}+p \Psi_{2 \beta}-\omega_{e l} \Psi_{2 \alpha} \\
U_{f \alpha}=R_{f} i_{f \alpha}+p \Psi_{f \alpha}+\omega_{e l} \Psi_{f \beta} \\
U_{f \beta}=R_{f} i_{f \beta}+p \Psi_{f \beta}-\omega_{e l} \Psi_{f \alpha} \\
\Psi_{A}=L_{1} i_{1 A}+\sqrt{\frac{2}{3}} L_{12}\left(i_{2 \alpha}+i_{f \alpha}\right) \\
\Psi_{B}=L_{1} i_{1 B}+\frac{1}{\sqrt{2}} L_{12}\left(i_{2 \beta}+i_{f \beta}\right)-\frac{1}{\sqrt{6}} L_{12}\left(i_{2 \alpha}+i_{f \alpha}\right) \\
\Psi_{C}=L_{1} i_{1 C}-\frac{1}{\sqrt{2}} L_{12}\left(i_{2 \beta}+i_{f \beta}\right)-\frac{1}{\sqrt{6}} L_{12}\left(i_{2 \alpha}+i_{f \alpha}\right) \\
\Psi_{2 \alpha}=L_{2} i_{2 \alpha}+L_{12}\left(\sqrt{\frac{3}{2}} \cdot i_{A}+i_{f \alpha}\right) \\
\Psi_{2 \beta}=L_{2} i_{2 \beta}+L_{12}\left(\frac{1}{\sqrt{2}} \cdot\left(i_{B}-i_{C}\right)+i_{f \beta}\right) \\
\Psi_{f \alpha}=L_{f} i_{f \alpha}+L_{12}\left(i_{2 \alpha}+\sqrt{\frac{3}{2}} \cdot i_{A}\right) \\
\Psi_{f \beta}=L_{f} i_{f \beta}+L_{12}\left(i_{2 \beta}+\frac{1}{\sqrt{2}} \cdot\left(i_{B}-i_{C}\right)\right) \\
\Delta \omega(t)=\frac{1}{2 J} \int_{0}^{t}\left(M_{i \dot{I} \tilde{O}}-M e_{e l}\right) d t \\
\omega_{e l}(t)=\Delta \omega(t)+\omega_{S}
\end{array}\right.
$$


Figure 4 provides the results of modelling by means of the proposed system of the SM supplying from AI taking into account saturation by magnetic lines which was set up by obtained a posteriori dependency of inductance on the current $\mathrm{L}=\mathrm{f}(\mathrm{I})$ with small value of the load coefficient for the SM.

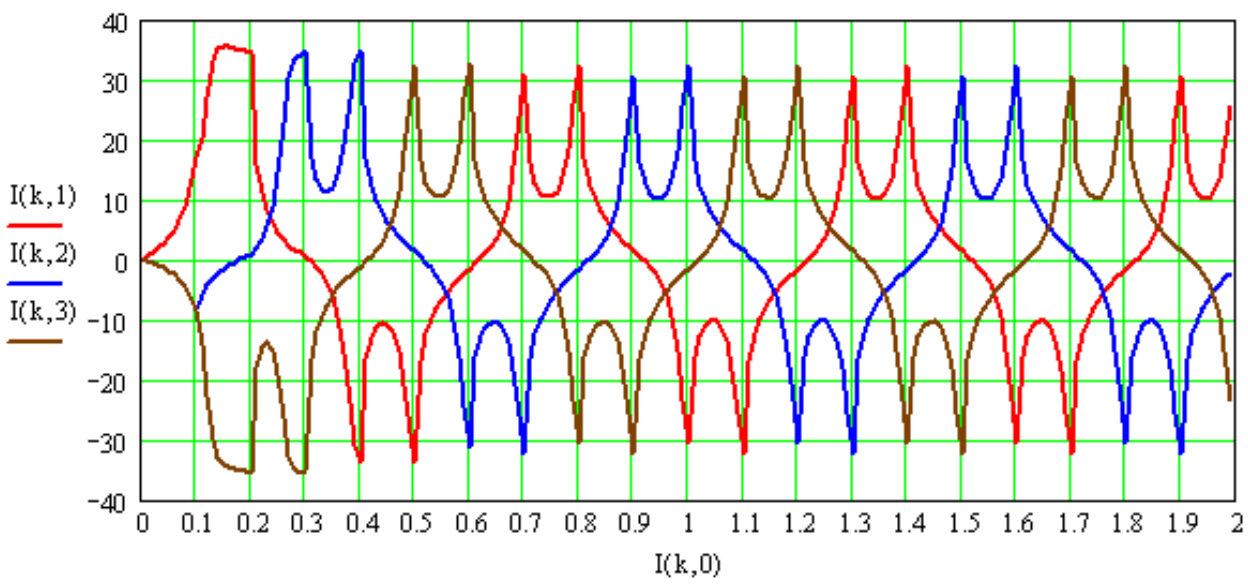

Figure 4. The result of the simulation, taking into account the current SM saturation

\section{Conclusions}

At present, the interest in application of synchronous machines in the various systems of the electric drive and energy sources is still growing. Synchronous motors and their modifications (ac converter-fed motor, etc.) enable to develop low-noise, reliable and economically efficient electric drive systems. They provide high maneuverability when using a propeller power plant of the submersible vehicles and the World fleet vessels. Synchronous motors are more and more widely implemented in the systems of the electric drive in pump, compressor and air-conditioning plants, in particular, in the oil and gas extracting industry. Synchronous generators are the major energy sources in the electric power systems of the variety autonomous plants: on vessels, offshore and coastal oil rigs, etc. Nowadays with the growing complexity of the automation systems, the interest in the problem of stability of synchronous machines performance is picking up. Great attention is paid to such a developing type of synchronous machines as asynchronized synchronous machines and synchronous generators of low capacity. The reason for the deepening interest in synchronous machines is rapid development of the systems of semiconducting converters, microprocessor units and a digital electric device.

The research conducted in this paper is applied in the calculation of reliability of work drilling platforms and ferry.

Proposed mathematical description of the SM is based on the modelling in stator winding axis, i.e. using true variables of the stator for the three-phase machine in axes A-B-C. The model makes possible as initial data to apply catalogue data for the SM designed for its being described in rotating axes. It enables to reject the transition to the rotating axes and while describing PPE switched to the SM. Thus, PPE, including its actuating system, when using the model proposed, may be described by equations of the variable of the state on the account of the description of the functions of switching to the temporary field. It enables to reject the methods of formation of actuating signals in the frequency field that as a rule was required when modelling the SM by Park-Gorev's equations.

\section{References}

1. Bolotin, B.I. and Vainer, V.L. (1974) Engineering methods for calculation of stability of ship's automated systems. Shipbuilding, $332 \mathrm{p}$.

2. Chernyi, S. and Zhilenkov, A. (2015) Modeling of Complex Structures for the Ship's Power Complex Using Xilinx System. Transport and Telecommunication Journal, 16(1), pp. 73-82. DOI: 10.1515/ttj2015-0008.

3. Chernyi, S and Zhilenkov, A. (2015) Analysis of complex structures of marine systems with attraction methods of neural systems. Metallurgical and Mining Industry, 1, pp. 37-44. 
4. Govindarajan, S.N. et al. (1991) Survey of Harmonic Levels on the Southwestern Electric power Company System. IEEE tram. on Power Delivery, 6 (4), pp. 1869-1873.

5. IEEE Working Group on Power System Harmonic. (1984) Bibliography of Power System Harmonics, Part 1, 84WM 214-3.

6. IEEE Working Group on Power System Harmonic. (1983) Power System Harmonics: An Overview, XEEE Dans. on Power App. Syst., PAS-102 (8), pp. 2455-2460.

7. Manchur, G. and Erven, C. (1992) Development of a Mode1 for Predicting Flicker from Electric Are Fumaces. IEEE Tran. On Power Delivery, 1.7(1), pp. 416-426.

8. Konoplev, K.G. (2008) Impulse governing of synchronous generators. SevNTY, 258 p.

9. Konstantinov, V.N. (1988) Systems and devices for automation of the ship's power plants. Shipbuilding, $312 \mathrm{p}$.

10. Lokhanin, E.K., Glagolev, V.A. and Skrypnik, A.I. (2008) Modelling of synchronous machines. Collected reports of the $3^{\text {rd }}$ international scientific and practical conference "Energy system: management, conference, education", pp. 24-44.

11. Markovich, I.I. (1969) Regimes for power systems. Energy, 350 p.

12. Mikhalev, S.V. (2013) Mathematical model in order to assess stability of the synchronous electric motors with the short-term supply failure. Modern society, education and since: proceedings according to the materials International scientific and practical conference. Business-Science-Society, pp. 99103.

13. Singh, B., Al-Haddad, K. and Chandra A. (1999) A Review of Active Filters for Power Quality Improvements. IEEE Trans. on Indusinal Electronics, 46(5), pp. 960-971.

14. Tolshin, V.I., Bolotin, B.I. and Konx, G.A. (1969) Impact of fuel apparatuses on stability of dieselgenerator with the capacity $150 \mathrm{kWt}$. Proceedings of the scientific research institute of the information of the heavy machines, 4, pp. 3-9.

15. Zhilenkov, A. and Chernyi, S. (2015) Investigation performance of marine equipment with specialized information technology. Energy Procedia, 100, pp. 1247-1252. DOI: 10.1016/j.proeng.2015.01.490. 